\title{
High Resolution Computed Tomography (HRCT) Assessment of Suspected COVID-19 Patients -----A Study of 51 Patients
}

Dr. Md Ubaidul Islam ${ }^{*}$, Dr. Yeasir Ahmed Masum², Dr. Laila Rubaiat ${ }^{3}$, Dr. Sudipta Gope ${ }^{4}$, Dr. M.N. Rubaia Islam Bony $^{5}$, Dr. Justin Clump ${ }^{6}$, Dr. Mousumi Anuradha Sangma ${ }^{7}$

${ }^{1}$ Radiologist, Department of Radiology and Imaging, 250 Bed Hospitals, Moulvibazar, Bangladesh

${ }^{2}$ Radiologist, Department of Radiology and Imaging, Railway General Hospital, Dhaka, Bangladesh

${ }^{3}$ Consultant Radiologist, Department of Radiology and Imaging, 250 Bed Hospitals, Moulvibazar, Bangladesh

${ }^{4}$ Assistant Professor, Department of Radiology and Imaging, Sylhet M A G Osmani Medical College and Hospital, Sylhet, Bangladesh

${ }^{5}$ Radiologist, Department of Radiology and Imaging, Sheikh Hasina National Institute of Burn and Plastic Surgery, Dhaka, Bangladesh

${ }^{6}$ Radiologist, Department of Radiology and Imaging, Chittagong Medical College and Hospital, Chittagong, Bangladesh

${ }^{7}$ Consultant Radiologist, Department of Radiology and Imaging, Mymensingh Medical College and Hospital, Mymensingh, Bangladesh

DOI: $10.36347 /$ sjams.2020.v08i07.018

| Received: 08.07.2020 | Accepted: 16.07.2020 | Published: 21.07.2020

*Corresponding author: Dr. Md Ubaidul Islam

Abstract

Objective: To investigate High resolution computed tomography (HRCT) features of patients with suspected COVID 19 in Moulvibazar district, Bangladesh and to evaluate the diagnostic value of HRCT and reverse transcriptase polymerase chain reaction (RT PCR) for COVID 19 pneumonia. Methods: This retrospective study included all patients with COVID 19 pneumonia suspicion, who were examined by both HRCT and RT PCR at initial presentation. The sensitivities of both tests were then compared. For patients with a final confirmed diagnosis, clinical and laboratory data, in addition to HRCT imaging findings were evaluated. Results: Total of 21 cases was finally diagnosed with COVID-19 pneumonia among 51 suspected patients. Twenty patients had abnormal HRCT findings at presentation, and only one patient had a normal thoracic HRCT. Using RT-PCR, 19 cases showed positivity, with 2 cases initially missed. Amongst the missed cases, patients had a positive result in the second RT-PCR test (after 2 and 4 days) Therefore, sensitivity of HRCT examinations was $95.45 \%$ at presentation, whereas first round RT-PCR sensitivity was $86.36 \%$. Conclusion: COVID 19 manifest with chest HRCT imaging abnormalities even in asymptomatic patients. RT PCR may produce initial false negative results. We suggest that patients with typical HRCT findings with negative RT PCR results should be isolated, and RT PCR should be repeated to avoid misdiagnosis.

Keywords: Computed tomography, corona virus, respiratory syndrome, COVID-19.

Copyright @ 2020: This is an open-access article distributed under the terms of the Creative Commons Attribution license which permits unrestricted use, distribution, and reproduction in any medium for non-commercial use (NonCommercial, or CC-BY-NC) provided the original author and source are credited.

\section{INTRODUCTION}

Since December 2019, a succession of cases of pneumonia with unknown causes has appeared in Wuhan, Hubei Province, China. On January 7, 2020, the 2019 novel coronavirus was identified as the causative agent based on virus typing $[1,2]$. This is the seventh known coronavirus to infect humans. Two other notable examples include severe acute respiratory syndrome and Middle East respiratory syndrome, the former of which began in southern China and resulted in 774 deaths in 8098 infected individuals in 29 countries from November 2002 through July 2003, and the latter of which originated in Saudi Arabia and was responsible for 848 deaths among 2458(2).

The source of the infection was a novel coronavirus (SARS CoV2), with symptomatic and asymptomatic infections reported. The incubation period of the disease is generally 3-7 days, but no longer than 14 days. Due to its strong infectivity profile, early diagnosis and treatment are crucial; otherwise human mediated disease spread can seriously endanger public health [2].

Outbreaks in different countries prompted the Ministry of Health and Family Welfare (MOHFW) Bangladesh to equip its PCR (Polymerase chain reaction) lab at IEDCR (Institute of Epidemiology, Disease Control, and Research) for testing samples from suspected COVID-19 cases for 2019-nCoV. The first confirmed cases of COVID-19 Bangladesh were reported on 8 March, 2020 [3-5].

As the disease presents with atypical or organizing pneumonia often with a bilateral, peripheral, 
and basal predominant distribution, Non contrast chest HRCT can also be considered for early diagnosis of the disease. Chest non contrast HRCT demonstrates typical radiographic features in almost all COVID-19 patients, including ground-glass opacities, multifocal patchy consolidation, and/or interstitial changes with a peripheral distribution [5,6]. A contrast scan should not be preferred as contrast may impact the interpretation of ground-glass opacification (GGO) pattern. These typical features were also observed in patients with negative RT-PCR results but positive clinical symptoms [6-13].

Although viral nucleic acid detection using RT-PCR remains the standard of reference. However, with limitations of sample collection and transportation, and kit performance, the total positive rate of RT-PCR for throat swab samples was reported to be about $30 \%$ to $60 \%$ at initial presentation. In this situation, the low sensitivity of RT-PCR implies that many COVID-19 patients may not be identified and may not receive appropriate treatment in time [14-19]. Those patients constitute a risk for infecting a larger population given the highly contagious nature of the virus.

It has been noted in small-scale studies that the current RT-PCR testing has limited sensitivity, while chest HRCT may reveal pulmonary abnormalities consistent with COVID-19 in patients with initial negative RT-PCR results.

\section{Materials ANd Methods Participants}

The retrospective study was approved by the institutional review board of Lifeline Hospital and 250 Bed District Hospital, Moulvibazar. This study was done in Lifeline Hospital and cardiac centre at Moulvibazar district of Bangladesh. Signed informed consent was exempted due to the retrospective nature of the study. Inclusion criteria were as follows: (a) Patients with a fever of > 38 and COVID-19 pneumonia suspicion (b) Patients with throat pain cough and chest pain (c) Patient with contact history and mild symptoms. (d) who underwent both thin-section HRCT of the chest and RT-PCR examinations. Exclusion criteria: (a) patients free from symptoms (b) patients transferred to another hospital or lost to follow-up. From 20 April, 2020 to 13 July, 2020, a total of 51 patients suspected for COVID-19 underwent chest HRCT examinations. The 51 patients underwent both HRCT and RT-PCR. HRCT was done in Lifeline hospital and RT PCR was held in 250 bed district hospital, Moulvibazar. The gold standard for a final diagnosis was positivity of first or repeated RT-PCR tests. Amongst the 51 included cases, 21 patients were finally diagnosed with COVID-19 pneumonia.

The other 30 patients without COVID-19 pneumonia served as the control group (Fig. 1)

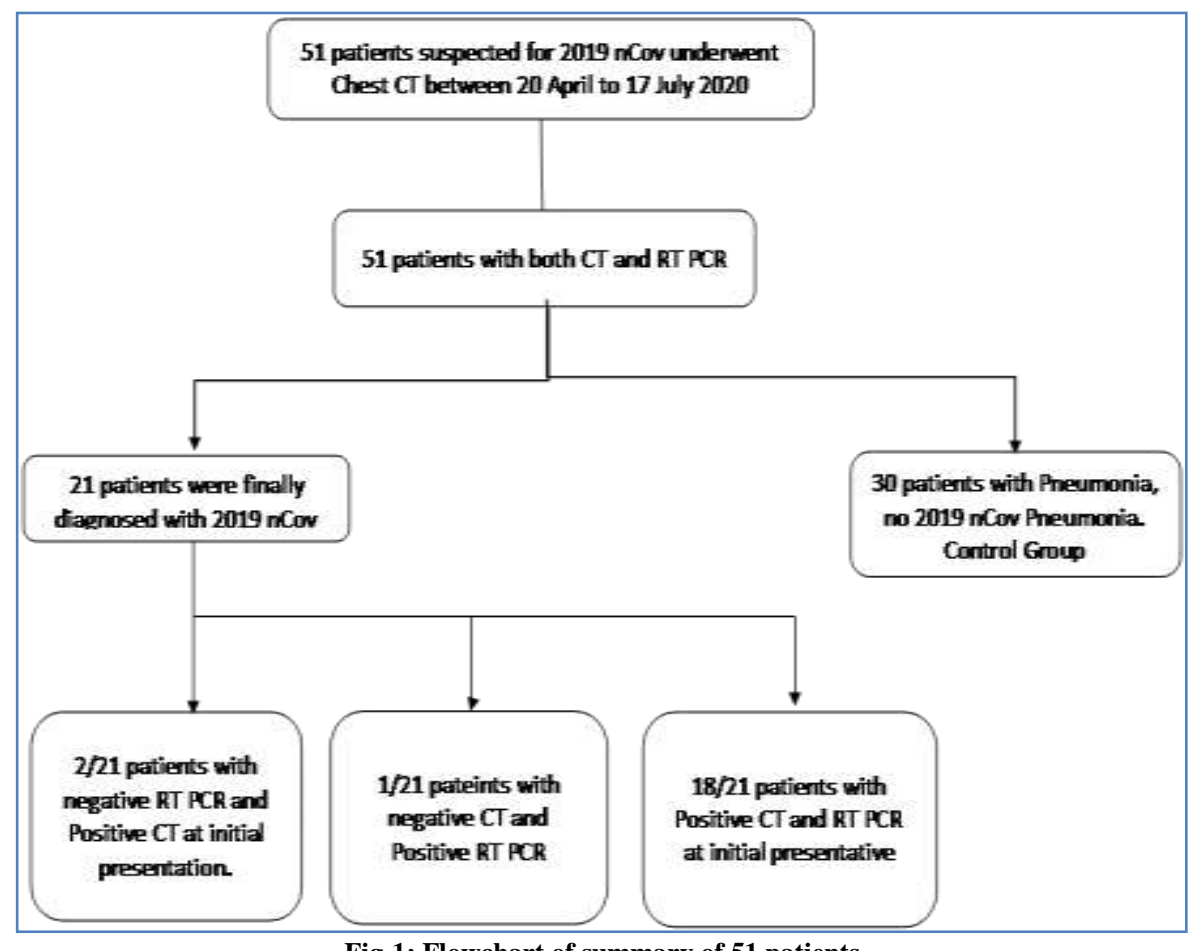

Fig-1: Flowchart of summary of 51 patients

\section{Image acquisition}

CT examinations were performed on a 64section scanner (GE Healthcare). The scanning parameters were as follows: $120 \mathrm{kV}$; 250mAs:; rotation time, 0.35 second; pitch, 1.5. Images were reconstructed with a $2 \mathrm{~mm}$ slice thickness, using a high frequency reconstruction algorithm. Acquisitions were performed during a deep inspiration breath-hold, without contrast 
administration. In cases of disagreement, a consensus was reached. CT evaluations included thelobar location and pattern of the lesion. In addition, the outer $1 / 3$ of the lung field was defined as a peripheral distribution, whereas the remainder was defined as a central distribution. In terms of pattern, ground-glass opacity (GGO) was defined as a modest increase in lung attenuation on lung window CT images, not obscuring the pulmonary vessels. Consolidation was defined as high density patchy opacities, inside which air bronchogram(s) could be observed. Lymphadenopathy was defined as a lymph node $>1.0 \mathrm{~cm}$ in short-axis diameter.

\section{CT staging ------}

In stage 1 (early stage, 0-4 days after the onset of initial symptoms), GGO was the main radiologic demonstration.

In stage 2 (progressive stage, 5-8 days after the onset of initial symptoms), the infection rapidly aggravated and extended to a bilateral multilobe distribution with diffuse GGO, crazy-paving pattern, and consolidation.

Table-1: Demographic and clinical characteristics of the 51 patients suspected with COVID-19 Pneumonia $(\mathrm{x} \pm \mathrm{s})$

\begin{tabular}{|l|l|l|l|}
\hline \multicolumn{1}{|c|}{ Variable } & $\begin{array}{c}\text { COVID-19 } \\
(\mathbf{n = 2 1})\end{array}$ & $\begin{array}{c}\text { Control Group } \\
(\mathbf{n = 3 0})\end{array}$ & $\boldsymbol{P}$ \\
\hline Gender & & & \\
\hline emale & 6 & 8 & 0.88 \\
\hline Male & 15 & 22 & \\
\hline Age (year) & $44.95 \pm 14.9$ & $45.46 \pm 11.9$ & 0.89 \\
\hline Exposure history & $19(90.5 \%)$ & $17(56.7 \%)$ & 0.09 \\
\hline Duration of Illness (Mean SD) & $5.0 \pm 2.3$ & $3.3 \pm 1.6$ & 0.006 \\
\hline Leukocyte count (normal or decreased) & $15(71.4 \%)$ & $10(33.3 \%)$ & 0.007 \\
\hline Lymphocytes (decreased) & $13(61.9 \%)$ & $8(26.7 \%)$ & 0.012 \\
\hline Fasting Glucose (increased) & $9(42.9 \%)$ & $5(16.7 \%)$ & 0.039 \\
\hline
\end{tabular}

Table 1: Shown demographic and clinical characteristics of the 51 patients

Exposure history was defined as having been exposed to infected patients within two weeks. Normal leukocyte counts $(4.0-10.0) \times 10^{9} / \mathrm{L}$, normal percentage of lymphocytes $20 \%-50 \%$, normal fasting glucose level: 3.9-6.1 mmol/L.

There were no significant differences in terms of gender, age, and time from fever to visit between the COVID-19 pneumonia group and control group. The exposure history of the COVID-19 pneumonia group exceeded that of the control group $(\mathrm{P}<0.05)$. Regarding laboratory examinations, the proportions of normal or decreased leukocyte counts and decreased lymphocytes in the COVID-19 group were higher than those of the control group $(\mathrm{P}<0.05)$. Additionally, the percentage of increased fasting glucose was higher in the infected vs. control group $(42.9 \%$ vs $16.7 \%, \mathrm{P}=$ 0.039).
In stage 3 (peak stage, 9-13 days after the onset of the initial symptoms), the involved area of the lungs slowly increased to peak involvement and dense consolidation became more prevalent. Findings included diffuse GGO, crazy-paving pattern, consolidation, and residual parenchymal bands.

In stage 4 (absorption stage, 14 days after the onset of initial symptoms), the infection was controlled and the consolidation was gradually absorbed. However, in this process, extensive GGO could be observed as the demonstration of the consolidation absorption.

\section{Statistical Analysis}

$\mathrm{Z}$ compared using a chi-square test. $\mathrm{P}<0.05$ was considered statistically significant.

\section{RESULTS}

Clinical and laboratory findings 
2). In patients without COVID-19 pneumonia, lower lobes distribution was more common than upper lungs location (Table 2). The occurrence of GGO or GGO with consolidation was more frequent in the COVID-19 pneumonia group, whereas the occurrence of consolidation was more common in the non-COVID-19 pneumonia group $(\mathrm{P}<0.05)$. Only one patient $(4.76 \%)$ had lymphadenopathy and none of patients had pleural effusion in the COVID-19 pneumonia group.

Table-2: Shown HRCT Imaging findings in the 51 patients suspected with COVID-19 pneumonia

\begin{tabular}{|c|c|c|c|}
\hline Group & $\begin{array}{c}\text { COVID-19 pneumonia } \\
(n=21)\end{array}$ & $\begin{array}{c}\text { Control Group } \\
\mathbf{n}=\mathbf{3 0}\end{array}$ & $\boldsymbol{P}$ \\
\hline \multicolumn{4}{|l|}{ Distribution of the lesions } \\
\hline Left upper lobe & $8 / 21(38.1 \%)$ & $6 / 30(20.0 \%)$ & 0.15 \\
\hline Left lower lobe & $13 / 21(61.9 \%)$ & $18 / 30(60.0 \%)$ & 0.89 \\
\hline Right upper lobe & $9 / 21(42.9 \%)$ & $13 / 30(3.3 \%)$ & 0.97 \\
\hline Right middle lobe & $3 / 21(14.3 \%)$ & $9 / 30(30.0 \%)$ & 0.19 \\
\hline Right lower lobe & $15 / 21(71.4 \%)$ & $16 / 30(53.3 \%)$ & 0.19 \\
\hline Lesion in Peripheral area & $18 / 21(85.7 \%)$ & $9 / 30(30.0 \%)$ & 0 \\
\hline Lesion in central area & $3 / 21(14.3 \%)$ & $21 / 30(70.0 \%)$ & $\overline{0}$ \\
\hline Number of lesion (multiple/single) & $18: 3(6: 1)$ & $20: 10(2: 1)$ & 0.12 \\
\hline \multicolumn{4}{|l|}{ Pattern of the Lesions } \\
\hline Ground glass opacity (GGO) & $15 / 21(57.1 \%)$ & $6 / 30(20.0 \%)$ & 0.006 \\
\hline Consolidation & $4 / 21(19.0 \%)$ & $12 / 30(40.0 \%)$ & 0.11 \\
\hline GGO with consolidation & $11: 10$ & $14: 16$ & 0.69 \\
\hline Lymphadenopathy & $2 / 21(9.5 \%)$ & $3 / 30(10.0 \%)$ & 0.95 \\
\hline Pleural effusion & 0 & $4 / 30(13.3 \%)$ & 0.13 \\
\hline
\end{tabular}

Table-2: HRCT imaging findings in the 51 patients suspected with COVID-19 pneumonia
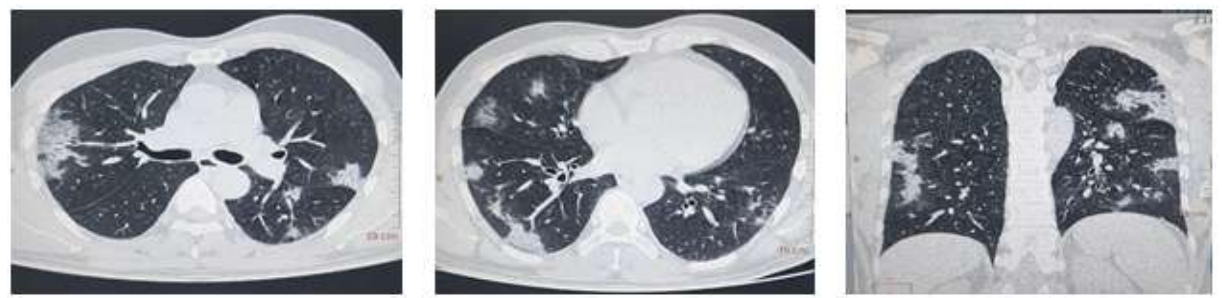

Fig-2: A 62 year old male patient with covid-19 pneumonia. HRCT of chest shows multifocal ground glass opacities with forming consolidations in both lungs having peripheral and random distribution.
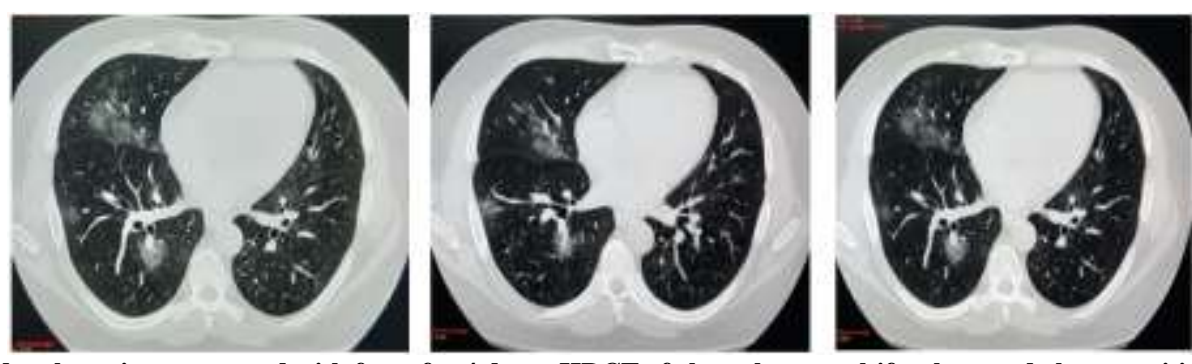

Fig-3: A 52 year old male patient presented with fever for 4 days. HRCT of chest shows multifocal ground glass opacities in both lungs with widening of vessels.
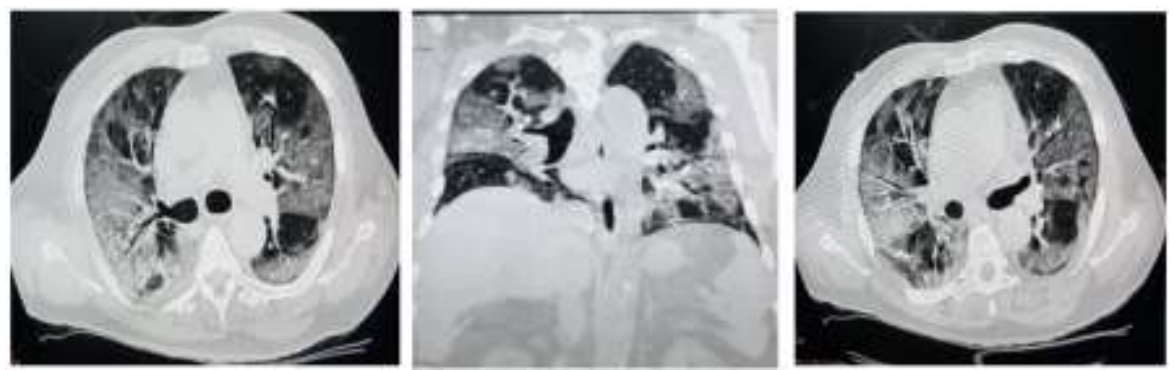

Fig-4: A 58 year old male patient presented with severe respiratory distress. HRCT of chest shows ground glass opacities with mosaic attenuation and areas of consolidation in both lungs. 

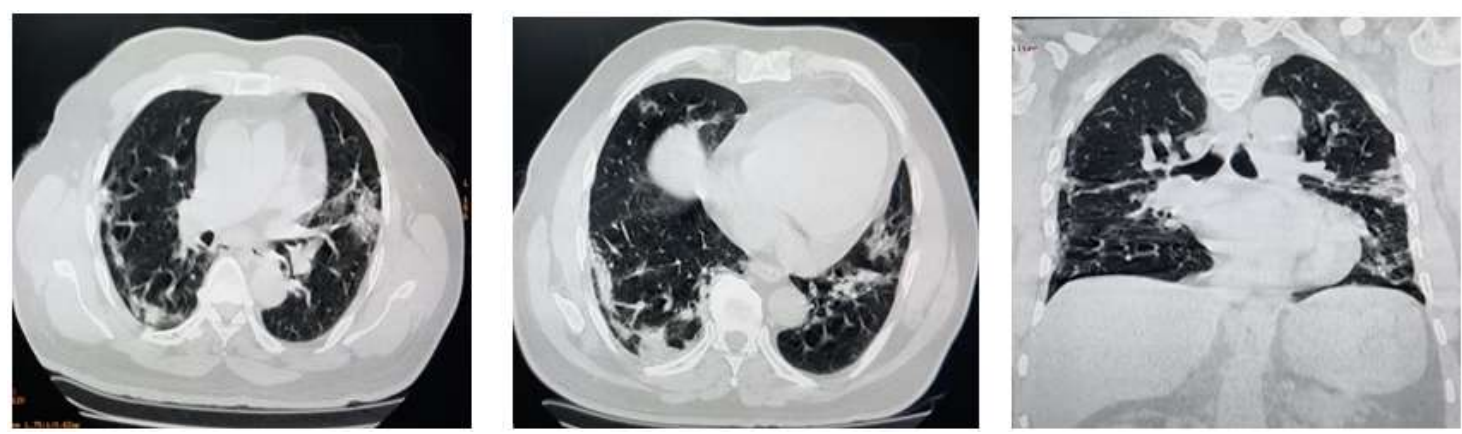

Fig-5: A 40 year old male patient presented with chest pain. HRCT of chest shows bilateral patchy areas of consolidation, ground glass opacities and sub pleural bands.

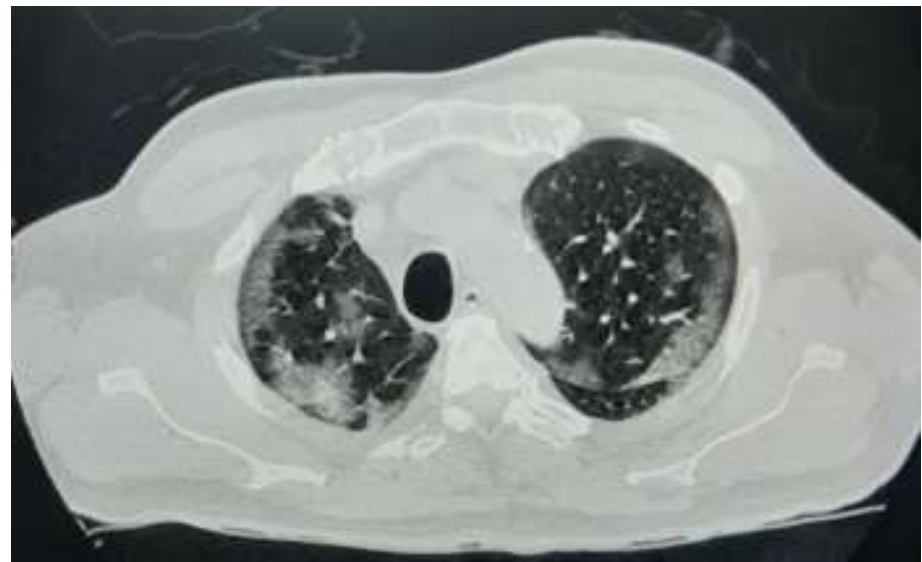

Fig-6: A 54-year male patient presented with fever and cough. HRCT chest showed crazy paving pattern in both lungs

\section{Comparison of the diagnostic efficacy between HRCT and RT-PCR}

A total of 21 cases were finally diagnosed with COVID-19 pneumonia. Twenty patients had abnormal HRCT findings at presentation, and only one patient had a normal chest HRCT. Using RT-PCR, 19 cases showed positivity, with 2 cases initially missed. Amongst the missed cases, patients had a positive result in the second RT-PCR test (after 2 and 4 days)) (Fig. 3). Therefore, sensitivity of HRCT examinations was 95.45 $\%$ at presentation, whereas first round RT-PCR sensitivity was $86.36 \%$.

\section{DISCUSSION}

An outbreak of coronavirus disease 2019 (COVID-19) infection began in December 2019 in Wuhan, the capital of central China's Hubei province. The pneumonia epidemic caused by a novel coronavirus was issued as a public health emergency of international concern by the WHO $[1,2]$.

For patient with COVID pneumonia currently affected with moderate to severe symptoms WHO suggest chest imaging in addition to others data to improve the therapeutic management. A rapid advice guide by world health organization (WHO) published on June 2020 recommended for the use of chest imaging in acute care of adult patient with suspected probable or confirmed COVID -19 including chest radiography and CT scan. HRCT chest is an important tool for both diagnosis and management of COVID -19 patients as well as it is an important complement to RTPCR test $[2,20]$.

The purpose of this study was to compare the sensitivity of HRCT and viral nucleic acid assay at initial presentation. In the absence of specific therapeutic drugs or vaccine it is essential to detect the disease at an early stage and immediately isolate an infected person from healthy population [21].

Our retrospective analysis showed that the sensitivity of initial HRCT was $95.45 \%$, whereas initial RT-PCR sensitivity was $86.36 \%$, with 2 initially missed cases which was almost similar from a report from European Journal of Radiology [17]. This may be related to sample collection as pharyngeal oral and nasal sampling are easier collection methods, whereas lower respiratory tract sampling is relatively difficult to perform, with medical staff susceptible to get infected $[10,22]$.

Chung reported that 3 patients diagnosed with COVID-19 pneumonia showed normal CT findings [23]. In our study, only one patient was observed with positive RT-PCR but negative HRCT. Considering that the results of RT-PCR may be false-negative, and the relatively long assay time, we recommend that the patients with typical imaging findings should be isolated and RT-PCR repeated to avoid misdiagnosis. 
The total number of leukocytes and lymphocytes in COVID 19 group were lower than those of control which were similar to previous reported in the literature [4-6]. Interestingly, we found that more patients with increased blood glucose levels were observed in the COVID-19 pneumonia group as compared to the control group (47\% vs $16.7 \%$ ), although the difference was not statistically significant $(P=0.039)$.

Common symptoms of patients are fever, cough and respiratory distress which are consistent with reports from various countries. In our study from Bangladesh also found fever and cough as common presenting symptoms [4].

The typical imaging features of COVID-19 pneumonia consist in single or multiple patchy consolidations or GGO in both lungs. In this study, GGO and GGO with consolidation were more frequent with $57.1 \%, 11.10 \%$ respectively [23]. Regarding distribution of lesions right lower lobe was most commonly affected $(71.4 \%)$ followed by left lower lobe $(61.9 \%)$. Compared with control group distribution were mostly peripheral with random distribution in all lobes [24]. Pleural effusion and lymphadenopathy were rarely observed, consistent with previous studies.

This study had some limitations. First there were incomplete histories, some patients took various drugs that may alter the HRCT findings additionally. Clinically suspected COVID 19 patients with negative RT PCR but positive HRCT findings refused to do RT PCR once again. The sample size of this study was small.

In summary, Initial RT-PCR and chest HRCT had comparable diagnostic performance in identification of suspected COVID-19 patients outside the epidemic centre. To compensate potential risk of false-negative RTPCR, chest HRCT should be applied for clinically suspected patients with negative initial RT-PCR.

\section{CONCLUSiON}

Patients with COVID 19 pneumonia present with typical abnormal signs on HRCT which has a higher diagnostic sensitivity. Sensitivity of RT-PCR is less than optimal. So the combination of clinical symptoms, exposure history, and typical HRCT features must be considered to identify COVID-19 cases.

\section{REFERENCES}

1. Zhu N, Zhang D, Wang W, Li X, Yang B, Song J, Zhao X, Huang B, Shi W, Lu R, Niu P. A novel coronavirus from patients with pneumonia in China, 2019. New England Journal of Medicine. 2020 Jan 24.
2. Chen Y, Liu Q, Guo D. Emerging coronaviruses: genome structure, replication, and pathogenesis. Journal of medical virology. 2020 Apr;92(4):41823.

3. Hossain I, Khan MH, Rahman MS, Mullick AR, Aktaruzzaman MM. The epidemiological characteristics of an outbreak of 2019 novel coronavirus diseases (COVID-19) in Bangladesh: A descriptive study. Journal of Medical Science and Clinical Research. 2020;8(04).

4. Zhao S, Lin Q, Ran J, Musa SS, Yang G, Wang W, Lou Y, Gao D, Yang L, He D, Wang MH. Preliminary estimation of the basic reproduction number of novel coronavirus (2019-nCoV) in China, from 2019 to 2020: A data-driven analysis in the early phase of the outbreak. International journal of infectious diseases. 2020 Mar 1;92:2147.

5. Wang C, Horby PW, Hayden FG, Gao GF. A novel coronavirus outbreak of global health concern. The Lancet. 2020 Feb 15;395(10223):470-3.

6. Lancet T. Emerging understandings of 2019-nCoV. Lancet (London, England). 2020 Feb 1;395(10221):311.

7. Lu H, Stratton CW, Tang YW. Outbreak of pneumonia of unknown etiology in Wuhan, China: The mystery and the miracle. Journal of medical virology. 2020 Apr;92(4):401-2.

8. Ajlan AM, Ahyad RA, Jamjoom LG, Alharthy A, Madani TA. Middle East respiratory syndrome coronavirus (MERS-CoV) infection: chest CT findings. American journal of roentgenology. 2014 Oct;203(4):782-7.

9. Chan JF, Yuan S, Kok KH, To KK, Chu H, Yang J, Xing F, Liu J, Yip CC, Poon RW, Tsoi HW. A familial cluster of pneumonia associated with the 2019 novel coronavirus indicating person-to-person transmission: a study of a family cluster. The Lancet. 2020 Feb 15;395(10223):514-23.

10. Zhou P, Yang XL, Wang XG, Hu B, Zhang L, Zhang W, Si HR, Zhu Y, Li B, Huang CL, Chen HD. A pneumonia outbreak associated with a new coronavirus of probable bat origin. nature. 2020 Mar;579(7798):270-3.

11. Perlman S. Another decade, another coronavirus. N Engl J Med.2020; 382(8): 760-762.

12. Wang Q, Zhang Z, Shi Y, Jiang Y. Emerging H7N9 influenza A (novel reassortant avian-origin) pneumonia: radiologic findings. Radiology. 2013 Sep;268(3):882-9.

13. Koo HJ, Lim S, Choe J, Choi SH, Sung H, Do KH. Radiographic and CT features of viral pneumonia. Radiographics. 2018 May;38(3):719-39.

14. Xu Z, Shi L, Wang Y, Zhang J, Huang L, Zhang C, Liu S, Zhao P, Liu H, Zhu L, Tai Y. Pathological findings of COVID-19 associated with acute respiratory distress syndrome. The Lancet respiratory medicine. 2020 Apr 1;8(4):420-2.

15. Tian S, Hu W, Niu L, Liu H, Xu H, Xiao SY. Pulmonary pathology of early phase 2019 novel 
coronavirus (COVID-19) pneumonia in two patients with lung cancer. Journal of Thoracic Oncology. 2020 Feb 28.

16. Tian HY. 2019-nCoV: new challenges from coronavirus. Zhonghua yu fang yi xue za zhi [Chinese journal of preventive medicine]. $2020 \mathrm{Feb}$ 3;54:E001-.

17. Long C, Xu H, Shen Q, Zhang X, Fan B, Wang C, Zeng B, Li Z, Li X, Li H. Diagnosis of the Coronavirus disease (COVID-19): rRT-PCR or CT?. European journal of radiology. 2020 Mar 25:108961.

18. Zhao S, Lin Q, Ran J, Musa SS, Yang G, Wang W, Lou Y, Gao D, Yang L, He D, Wang MH. Preliminary estimation of the basic reproduction number of novel coronavirus (2019-nCoV) in China, from 2019 to 2020: A data-driven analysis in the early phase of the outbreak. International journal of infectious diseases. 2020 Mar 1;92:2147.

19. Zhang N, Wang L, Deng X, Liang R, Su M, He C, $\mathrm{Hu}$ L, Su Y, Ren J, Yu F, Du L. Recent advances in the detection of respiratory virus infection in humans. Journal of medical virology. 2020 Apr;92(4):408-17.

20. Lei J, Li J, Li X, Qi X. CT imaging of the 2019 novel coronavirus (2019-nCoV) pneumonia. Radiology. 2020 Apr;295(1):18

21. Kanne JP. Chest CT findings in 2019 novel coronavirus (2019-nCoV) infections from Wuhan, China: key points for the radiologist.

22. Song F, Shi N, Shan F, Zhang Z, Shen J, Lu H, Ling Y, Jiang Y, Shi Y. Emerging 2019 novel coronavirus (2019-nCoV) pneumonia. Radiology. 2020 Apr;295(1):210-7.

23. Jiang Y, Guo D, Li C, Chen T, Li R. Highresolution CT features of the COVID-19 infection in Nanchong City: Initial and follow-up changes among different clinical types. Radiology of Infectious Diseases. 2020 May 13.

24. He X, Zheng J, Ren JL, Zheng G, Liu L. Chest high-resolution computed tomography imaging findings of coronavirus disease 2019 (COVID-19) pneumonia. International Journal of Radiation Research. 2020 Apr 1;18(2):343-9. 